\section{Drive and performance modification following shifts in the photoperiod}

FREDERICK H. ROHLES, JR., Institute for Environmental Research, Kansas State University, Manhattan, Kans. 66502

The effects of 12-h phase shifts in the light-dark cycle on feeding behavior and performance were studied by requiring a monkey to perform an 18-problem instrumental skill sequence for all of its food ad lib. The photoperiod of $12 \mathrm{~h}$ light/ $12 \mathrm{~h}$ dark was advanced by $12 \mathrm{~h}$ after 28 days and 28 days later was shifted back again. The results showed that almost all of the food was obtained during the light phase, and 5 days were required for the feeding behavior to stabilize during the "new" light phase following the initial phase shift of $12 \mathrm{~h}$; however, stabilization occurred after 3 days following the second shift. $A$ significant decrement in performance accuracy was observed on the 3rd and 4th days after the first phase shift and on the 2nd day after the return shift. Results are discussed in terms of the stress of photoperiodic loading.

Informal and subjective reports of intercontinental jet travelers emphasize feelings of fatigue and a general disorientation to the work, sleep, and eating activities of the "new" time zone. Problems such as these, which are closely associated with high-speed transmeridian aircraft travel and, to some extent, space travel, go beyond mere differences in "times of day" and are related to five factors that may be grouped under a stress condition we can identify as photoperiodic loading. The first of these factors is the length of the day-night or light-dark cycle; this differs, of course, according to the time of the year and the degrees above or below the equator. The phase angle difference is the second factor and refers to the difference between two corresponding phase angles either in degrees or in time zone units. Thus, the difference in the phase angle of the light-dark cycles of New York and Rome is $90 \mathrm{deg}$ or $6 \mathrm{~h}$ (both New York and Rome are approximately $40 \mathrm{deg}$ $\mathrm{N}$ latitude and hence have the same number of hours of daylight and darkness on any given day); and an individual traveling between these two cities is said to experience a $6 \cdot \mathrm{h}$ shift in the light-dark cycle. The fact that he may go from New York to Rome or vice versa demands an additional qualifier, namely, the direction of shift. Four combinations of directional shifts are possible when traveling from one meridian to another and retuming: (1) West-West, West from New York to Bangkok, a phase angle difference of $12 \mathrm{~h}$, and West from Bangkok to New York; (2) West-East, West from New York to Bangkok and East from Bangkok back to New York; (3) East-East, East from New York to Bangkok and East from Bangkok back to New York, and (4) East-West, East from New York to Bangkok and West from Bangkok on the return flight to New York. The fourth factor, known as reentrainment duration, or stopover time, is the number of days or hours the $S$ remains at the new time zone. The fifth factor contributing to photoperiodic loading is the rate that the $S$ experiences differences in the phase angle and displacements of $2-3 \mathrm{~h}$ in any $24 \mathrm{~h}$ period appear to have little or only minor effects. Investigation of photoperiodic loading was studied by Hauty and Adams who demonstrated that reaction time in human Ss increased significantly following a flight from Oklahoma City to Manila, P.I., a shift in the light-dark cycle of $10 \mathrm{~h}$. Similar findings were observed following a West-East flight from Oklahoma City to Rome, Italy, and again on the return flight, each a 6-h shift in the light-dark cycle; but beyond these field studies, little systematic research has been done.

In a different approach to studying behavior and the photoperiod, Farrer \& Ternes (1969) showed that barpressing behavior conformed to a circadian rhythm; however, Rohles (1967a) interpreted these results and those of his own similar findings to be measures of innate feeding rhythms and that barpressing was merely a convenient and effective tool for studying the rhythmical characteristics of the hunger drive. In view of these findings, coupled with those of Hauty and Adams, it appeared only logical to design a study that would examine learned and innate behavior simultaneously as a function of photoperiodic loading. Thus, the purpose of this study was to determine the effects of shifts in the light-dark cycle on the feeding rhythm when performance on a learned task was required to obtain food. SUBJECT

The $\mathbf{S}$ was a female stumptail monkey (Macaca speciosa) whose age at the beginning of the study was 34 months.

\section{APPARATUS}

The apparatus consisted of a $30 \times 30 \times 30$ in. test chamber that was equipped with three stimulus-response keys (Rohles, 1969) and a Gerbrands pellet dispenser for delivering $0.3 \mathrm{~g}$ CIBA food pellets. Water was available ad lib. The test chamber was housed in a double-wall isolation booth that was indirectly lighted by a $25-W$ incandescent lamp and a $7.5 \cdot \mathrm{W}$ "night light." The night light was illuminated continuously; the 2S-W lamp was turned on only during the "light phase" of the experiment. The test chamber and booth were housed in a climatic room whose temperature was maintained at $75^{\circ} \mathrm{F}$ throughout the study; the relative humidity was constant at $40 \%$. Programming of the task was automatic, with the correct and incorrect responses being recorded every $30 \mathrm{~min}$ on a digital printer.

\section{PROCEDURE}

The $S$ was trained on an instrumental skill sequence in which 18 three-stimulus oddity problems were presented in a fixed order (Rohles, 1961, 1967b). When these problems were solved correctly, the reward was delivered and the series of 18 problems was repeated again in the same order; incorrect discriminations were followed by a 5-sec "time-out." Asymptotic performance on this task is characterized by extremely rapid responding with very few errors, and when this occurred, the experiment was begun.

The $S$ was placed in the test chamber and isolation booth and was maintained in the climatic room for the next 84 days. The light-dark cycles during this period are shown in Table 1. During this time, the $S$ could obtain food any time it desired, but only by performing the 18-problem sequence. The booth was opened at random times once or twice a day for cleaning, checking the operation of the feeder and SRKs, and filling the food and water dispensers.

\section{RESULTS AND DISCUSSION}

The S's behavior during the 84-day period is shown in Fig. 1. Each $1 / 2$-h period in which the $S$ received one or more food pellets is indicated by a blackened square; and the mean daily accuracy on the performance task is indicated as a continuous record. With reference to the performance incidence, the most obvious result is that the animal obtained almost all of its food during the light periods. On Day 28, when the initial shift occurred, the $\mathrm{S}$ ate during the first $12 \mathrm{~h}$ but did not eat

Table 1

Light-Dark Periods by Days

\begin{tabular}{lcc}
\hline & \multicolumn{2}{c}{ Hour } \\
\cline { 2 - 3 } Day & $0800-2000$ & $2000-0800$ \\
\hline $1-27$ & Light & Dark \\
28 & Light & Light \\
$29-56$ & Dark & Light \\
57 & Dark & Dark \\
$58-84$ & Light & Dark \\
\hline
\end{tabular}




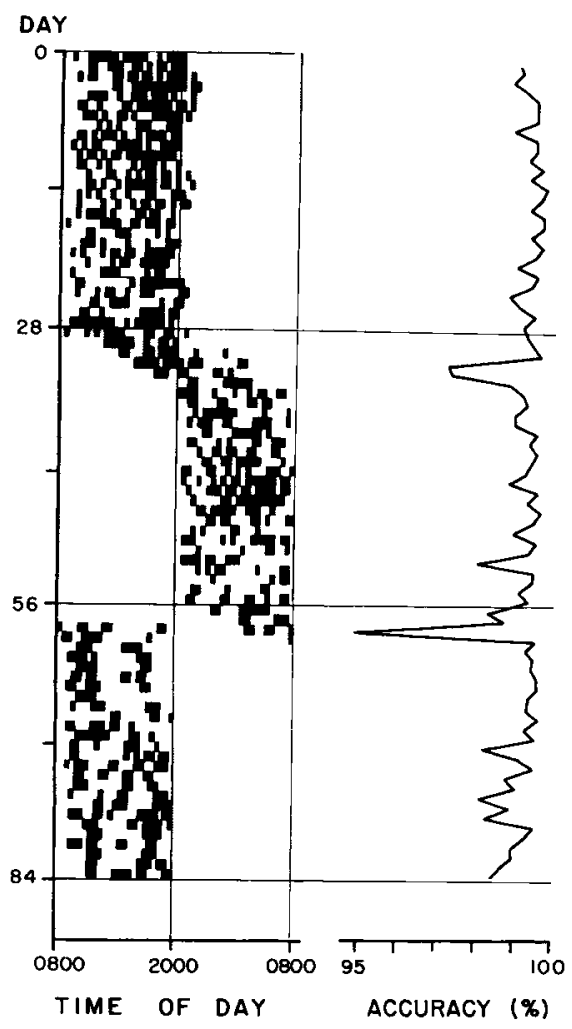

Fig. 1. Daily feeding incidence and performance accuracy (each blackened square represents one $1 / 2$-h period in which at least one reinforcement was obtained; light phase is white, dark phase is shaded).

during the second $12 \mathrm{~h}$, even though the light remained on. Then, on Days 29 through 33, a gradual shift in the feeding behavior occurred, and on Day 34 until Day 57, the day of the second shift, all of the feeding occurred during the light phase. On Day 57, the $S$ ate only during the second $12-\mathrm{h}$ period, which at that time had been changed to the dark condition. Similar feeding behavior was observed on Day 58; however, on Days 59 and 60, the behavior began to shift, and on Day 61 until the end of the study, the $S$ ate during the light phase only. Another interesting feature of the incidence of feeding is the apparent reduction in the frequency of feeding during the course of the study. In the first 28 days, the $S$ ate often but in short bursts; however, in the last 28 days, the feeding sessions lasted longer, but there were fewer of them. This behavior was accompanied by a high degree of accuracy during the first 28 days and, while the level of accuracy continued at a high level throughout the entire study, it became noticeably more variable as the study progressed.

The decrease in the performance accuracy following both shifts is greater than two standard deviations below the mean performance of the 28 days preceding the shift. Interestingly, these decrements did not occur on the days immediately following the shift, but occurred on the 3rd and 4th days following the initial shift and the 2 nd day after the return shift.

To consider these findings in photoperiodic loading terms, it must be concluded that following a 12 -h phase shift in a Westerly direction (Day 28, light $24 \mathrm{~h}$ ), feeding behavior requires 6 days (Day 34 ) to reentrain and and performance on a perceptual-motor skill requires 5 days to reentrain to its preshift level of accuracy; however, following a 12-h phase shift in an Easterly direction (Day 57, dark $24 \mathrm{~h}$ ) feeding behavior reentrains in 4 days (Day 61) and performance accuracy returns to its preshift level on the third day (Day 60) after the shift. And when these events occur around a 12-h-light/12-h-dark day, travel in a Westerly direction is more stressful than it is in an Easterly route. This finding agrees, in fact, with those of Hauty (1966a, b c) directly opposite to Wever's belief that "in flights to the antipodes (180 deg in 12 phase shifts) westward flights result in faster reentrainment
[Wever, 1966]." This difference, however, could be a result of the combined effects of the two shifts which might not have been considered individually. Nevertheless, they do present the complexity of the problem that can be answered only in a factorial design involving phase angle difference, direction of shift, and reentrainment time, but more important, they demonstrate conclusively the role of photoperiodic loading in drive modification and perceptual-motor skills performance.

\section{REFERENCES}

FARRER, D. N. \& TERNES, J. W. Illumination intensity and behavioral circadian rhythms. In F. Rohles (Ed.), Circadian rhythms in non-human primates. Basel: Karger, 1969. Pp. 1-7.

HAUTY, G. T., \& ADAMS, T. Phase shifts of the human circadian system and performance deficit during periods of transition: I. East-West flight. Aerospace Medicine, 1966a, 37, 668-674.

HAUTY, G. T., \& ADAMS, T. Phase shifts of the human circadian system and performance deficit during periods of transition: II. West-East flight. Aerospace Medicine, 1966b, 37, 1028-1033.

HAUTY, G. T., \& ADAMS, T. Phase shifts of the human circadian system and performance deficit during periods of transition: III. North-South flight. Aerospace Medicine, 1966c, 37, 1257-1262.

ROHLES, F. H. The development of an instrumental skill sequence in the chimpanzee. Joumal of the Experimental Analysis of Behavior, 1961, 4, 323-325.

ROHLES, F. H. Circadian thythms in the feeding behavior of laboratory monkeys. Laboratory Animals, 1967a, 1, 141-146.

ROHLES, F. H. Performance by a rhesus monkey in three randomly presented skill sequences. Folia Primatologica, 1967b, 7, 208-215.

ROHLES, F. H. Instrumentation for the operant conditioning experiment. American Psychologist, 1969, 24, 250-254.

WEVER, R. The duration of reentrainment of circadian rhythms after phase shifts of the Zeitgeber-A theoretical investigation. Journal of Theoretical Biology, 1966, 13, 187-201. NOTE

1. This research was supported in part by USAFOSR Contract F44620-68-C-0020. 